\title{
Towards Evidence-Based Weaning: a Mechanism-Based Pharmacometric Model to Characterize Iatrogenic Withdrawal Syndrome in Critically III Children
}

\author{
Sebastiaan C. Goulooze, ${ }^{1,2}$ Erwin Ista, ${ }^{3}$ Monique van Dijk,,${ }^{3,4}$ Dick Tibboel, ${ }^{3}$ \\ Elke H. J. Krekels, ${ }^{1}$ and Catherijne A. J. Knibbe ${ }^{1,5,6}{ }_{(D}$
}

Received 5 December 2020; accepted 22 March 2021; published online 17 May 2021

Abstract. For the management of iatrogenic withdrawal syndrome (IWS) in children, a
quantitative understanding of the dynamics of IWS of commonly used opioids and sedatives
is lacking. Here, we introduce a new mechanism-based pharmacokinetic-pharmacodynamic
(PKPD) modeling approach for studying IWS in pediatric clinical datasets. One thousand
seven hundred eighty-two NRS withdrawal scores of IWS severity were analyzed, which were $_{\text {collected from } 81 \text { children (age range: } 1 \text { month-18 years) that received opioids or sedatives }}$
by continuous infusion for 5 days or more. These data were successfully fitted with a PKPD
model consisting of a plasma and a dependence compartment that well characterized the
dynamics of IWS from morphine, fentanyl, and ketamine. The results suggest that (1) instead
of decreasing the infusion rate by a set percentage at set intervals, it would be better to
lengthen the weaning period when higher infusion rates are administered prior to weaning;
(2) for fentanyl specifically, the risk of IWS might be lower when weaning with smaller dose
reductions every 12 h instead of weaning with greater dose reductions every 48 h. The
developed PKPD model can be used to evaluate the risk of IWS over time and the extent to
which it is affected by different weaning strategies. The results yield hypotheses that could
guide future clinical research on optimal weaning strategies. The mechanism-based PKPD
modeling approach can be applied in other datasets to characterize the IWS dynamics of
other drugs used in pediatric intensive care.

KEY WORDS: Pharmacometrics; Pediatric; Iatrogenic withdrawal; Morphine; Fentanyl.

\section{INTRODUCTION}

Opioids and sedatives are crucial in ensuring critically ill children's well-being and comfort (1). Even though prolonged treatment may be required for clinical reasons, drug dependency and iatrogenic withdrawal syndrome (IWS) may occur during weaning of these drugs and subsequently hamper patient recovery $(2,3)$. Effective weaning strategies are crucial to prevent IWS itself but may also reduce situations where concerns about IWS lead to the undertreatment of pain and distress in critically ill children $(1,4)$.

\footnotetext{
${ }^{1}$ Division of Systems Biomedicine and Pharmacology, Leiden Academic Centre for Drug Research, Leiden University, Einsteinweg 55, 2333, CCLeiden, The Netherlands.

${ }^{2}$ LAP\&P Consultants BV, Leiden, The Netherlands.

${ }^{3}$ Pediatric Surgery, Erasmus Medical Center-Sophia Children's Hospital, Rotterdam, The Netherlands.

${ }^{4}$ Division of Nursing Science, Department of Internal Medicine, Erasmus Medical Center, Rotterdam, The Netherlands.

${ }^{5}$ Department of Clinical Pharmacy, St. Antonius Hospital, Nieuwegein, The Netherlands.

${ }^{6}$ To whom correspondence should be addressed. (e-mail: c.knibbe@antoniusziekenhuis.nl)
}

The reported incidence of IWS in different studies varies widely (5 to $87 \%$ ) (3). Prolonged treatment and high cumulative drug doses are the most commonly reported risk factors for IWS $(1,3)$. While risk factors can identify patients who need weaning, they do not inform us how weaning should be performed. Thus, different institutions apply a large variety of weaning strategies $(5,6)$. To reduce the risk of IWS in clinical practice, we need weaning strategies based on a patient's characteristics and type of drug and dosing history over time.

Population pharmacokinetic-pharmacodynamic (PKPD) modeling approaches could help to develop weaning strategies for IWS. In general, PKPD models use dosing information to predict drug concentrations over time and then relate these concentrations to the drug's effect (7). In this case, the effect would be an adverse effect-the occurrence of IWS. Rather than treating the risk of IWS as a static percentage, a PKPD modeling approach treats it as a dynamic risk that can change over time and is affected by the treatment before and during weaning. More specifically, these models can be used to simulate the risk of IWS over time in different scenarios, based on an individual's medication history-consisting of different opioids and sedatives in different doses and durations - and other 
patient-level risk factors. On the basis of this risk of IWS over time, different weaning strategies can be compared, and the most appropriate strategy can be selected.

In the underlying mechanism of IWS, drug treatment plays a dual role. Prolonged drug treatment may cause dependence, which puts the patient at risk of IWS (8). In a drug-dependent patient, ongoing treatment with the drug will prevent the occurrence of IWS, while stopping drug treatment too fast will likely incite IWS $(1,6)$. There are currently no PKPD models that can characterize the development of dependence over time during prolonged drug treatment, as well as the risk of IWS and disappearance of dependence over time during the weaning phase.

In this work, we propose a novel, mechanism-based PKPD model that is able to describe how exposure to selected opioids and sedatives over time contributes to the development and disappearance of drug dependence and the risk of IWS over time. The model was developed using data from a previous study in which the Sophia Observation withdrawal Symptoms scale (SOS) was validated in a PICU population of children who received continuous infusions of opioids or sedatives for 5 days or more (9). We performed model simulations to generate new hypotheses for optimal weaning strategies.

\section{METHODS}

\section{Clinical Study}

We performed a secondary analysis on data from a previous prospective observational study by Ista et al. (9) that included children from term neonates up to children of 18 years of age who received opioids or sedatives by continuous infusion for 5 days or more. The study was approved by the local institutional review board, which waived the need for parental informed consent. The study considered children admitted to the ICU between March 2009 and September 2010 in the Erasmus MC-Sophia Children's Hospital. From the 154 children included in the original study, we excluded all infants younger than 1 month and those receiving extracorporeal membrane oxygenation treatment, due to a lack of literature information on the population pharmacokinetics of the investigated opioids and sedatives in these populations.

The measure of IWS severity used in the current study is the $\mathrm{NRS}_{\text {withdrawal }}$ score, a numeric rating scale used by a member of the nursing staff to rate the IWS severity, ranging from 0 (no IWS) to 10 (worst IWS possible). This rating considers both the observed withdrawal-associated symptoms, as well as contextual factors such as the (duration of) use of opioids and sedatives, and the possibility of co-occurring pain, under-sedation or delirium (9). $\mathrm{NRS}_{\text {withdrawal }}$ scores above 3 are considered to reflect IWS that requires pharmacological intervention. A total of $1782 \mathrm{NRS}_{\text {withdrawal }}$ scores were available for the development of the mechanism-based PKPD model for IWS.

\section{Mechanism-Based PKPD Model for Iatrogenic Withdrawal}

We first calculated, on the basis of the administered dosages to each individual subject, the plasma concentration over time profiles of the main opioids and sedatives (morphine, fentanyl, methadone, midazolam, lorazepam, clonidine, ketamine, and propofol) administered during the PICU stay. For these calculations, we used appropriate pediatric population pharmacokinetic models of these drugs that characterized the drug disposition and drug clearance in our patient population (10-20). The bioavailability and rate of absorption for extravascular routes of administration were taken from pediatric population pharmacokinetic models or, if these were not available, from non-compartmental analyses in children $(14,21-23)$ or analyses in adults (24-32). Supplemental Material 1 provides a detailed overview of the population PK models and relevant literature references. This document also contains the number of drug doses and the routes of administration for each of the opioids and sedatives considered.

To characterize the development and disappearance of drug dependence over time, a novel mechanism-based IWS modeling approach was developed. For each drug, the model contains a hypothetical "dependence compartment" which equilibrates with the drug's central pharmacokinetic (plasma) compartment with an estimated rate constant $k_{\mathrm{dep}}$. This constant controls the rate at which patients become dependent on a particular drug, as well as the rate at which patients become less drug-dependent after drug treatment is reduced or discontinued:

$\frac{d C_{\text {dependence }}}{d t}=k_{\text {dep }} *\left(C_{\text {plasma }}-C_{\text {dependence }}\right)$

where $C_{\text {dependence }}$ represents the hypothetical concentration that the patient is dependent on and $C_{\text {plasma }}$ is the drug concentration in plasma. The "concentration shortage," defined as the difference between $C_{\text {dependence }}$ and $C_{\text {plasma, }}$, was used to drive the IWS severity using a linear relationship (Eq. 2). Drugs were considered to have no effect on IWS when $C_{\text {dependence }} \leq C_{\text {plasma }}$. The IWS severity caused by a single drug j (Effect ${ }_{\mathrm{j}}$ ) was defined according to Eq. 2:

$$
\text { Effect }_{\mathrm{j}}= \begin{cases}\text { Slope }_{j} *\left(C_{\text {dependence }}-C_{\text {plasma }}\right), & \text { if } C_{\text {dependence }}>C_{\text {plasma }} \\ 0 & \text { if } C_{\text {dependence }} \leq C_{\text {plasma }}\end{cases}
$$

where Effect ${ }_{j}$ represents the contribution to IWS by drug $j$, and slope $_{j}$ is the estimated slope parameter for this particular drug. Because $C_{\text {dependence }}$ and $C_{\text {plasma }}$ change over time, the Effect $_{\mathrm{j}}$ also changes over time. Non-linear alternatives (such as the $E_{\max }$ function) to Eq. 2 were also tested. Overall IWS severity was modelled as additive effects of each drug included in the model (Eq. 2) to an estimated baseline:

$\lambda_{i}=$ Baseline $_{i} \cdot e^{\eta_{i}}+\sum_{j=1}^{\text {Ndrugs }}$ Effect $_{j}$

where $\lambda_{i}$ is the overall IWS severity of individual $i$ at a particular time; Baseline B $_{i}$ is the estimated baseline IWS severity of a typical individual; $\eta_{i}$ represents the post hoc estimate of how much the baseline IWS severity of individual $i$ deviates from the typical baseline; and Ndrugs is the number of drugs included in the IWS model. 
We also explored whether drugs used to manage IWS during weaning (e.g., clonidine) could lower the IWS severity caused by other drugs, whether drugs would influence the IWS caused by drugs from the same class (e.g., morphine lowering the fentanylassociated withdrawal or vice versa), and whether the development of tolerance (in addition to dependence) could be modelled with an additional compartment. The model equations for this are given in the Supplemental Material 2.

The method proposed by Plan et al. was used to relate the model-predicted $\lambda_{i}$ to the probability of observing a particular $\mathrm{NRS}_{\text {withdrawal }}$ score (33). This method uses a generalized truncated Poisson model to account for the fact that only integer values of $0-10$ are possible outcomes of the $\mathrm{NRS}_{\text {withdrawal }}$ scale. To account for the correlation between subsequent observations within an individual, the model included a Markovian inflation of the probability of observing $\mathrm{NRS}_{\text {withdrawal }}$ scores that are within two points of the previously observed $\mathrm{NRS}_{\text {withdrawal }}$ score. We estimated the probability inflation of having the same score when the previous score was zero $(\pi 010)$, the probability inflation of having the same score for different previous scores $(\pi 0 \mid \mathrm{x})$, and the probability inflation of having a score that is \pm 1 points or \pm 2 points away from the previous score $(\pi \pm 1, \pi \pm 2)$. The final model code is provided in Supplemental Material 3.

\section{PKPD Model Development and Evaluation}

During model development, PKPD models were fitted with NONMEM 7.3 using the Laplace conditional estimation method. For the final PKPD model, an expectation-only step of the Importance Sampling (IMP) method-using the final parameter estimates from the Laplace method - was performed to obtain a covariance matrix of the parameter estimates (34).

Model development started with a base model in which none of the administrated drugs affected IWS severity. To this base model, using stepwise model development, the effects of different drugs were added to the model according to the Eqs. $1-3$, to see if doing so would improve the fit of the observed $\mathrm{NRS}_{\text {withdrawal }}$ data. Effects of drugs that did not improve the fit of the data were not included. Competing models were compared using the objective function value for nested models or the Akaike information criterion. The patient characteristics age, sex, Pediatric Risk of Mortality Score III (PRISM III), and diagnosis category were tested as covariates for the inter-individual variability in baseline IWS severity (Eq. 3) using a stepwise forward inclusion approach and included in the model if this resulted in a significant $(p<0.01)$ reduction in the objective function value.

Two techniques were used to evaluate whether the goodness-of-fit of the model and its predictions, by comparing them to the observed $\mathrm{NRS}_{\text {withdrawal }}$ data. Mirror plots (35) were used to compare the predicted and observed frequencies for each possible $\mathrm{NRS}_{\text {withdrawal }}$ score, as well as the frequencies of specific transitions between consecutive observations. The expected $\mathrm{NRS}_{\text {withdrawal }}$ at a particular observation is calculated as:

Expected $N R S_{\text {withdrawal }}=\sum_{j=0}^{10} j \times p($ score $=j)$ where $p($ score $=j)$ is the individual post hoc model-predicted probability of observing an $\mathrm{NRS}_{\text {withdrawal }}$ score equal to $j$ at a particular observation. Additionally, residuals were calculated as the difference between the observed $\mathrm{NRS}_{\text {withdrawal }}$ score and the expected $\mathrm{NRS}_{\text {withdrawal }}$ score. The residuals were then plotted against time and versus the expected $\mathrm{NRS}_{\text {withdrawal }}$ to visually inspect these plots for the presence of trends that might be indicative of model misspecification.

Using the PKPD model, we simulated different scenarios to confirm that the model's behavior is in agreement with clinical knowledge on the dynamics of IWS: abrupt discontinuation of a continuous infusion increases the risk of IWS, but this risk can be reduced by slowly weaning off the continuous infusion $(1,6)$. To aid interpretation of the simulation results, we calculated the probability of having an $\mathrm{NRS}_{\text {withdrawal }}$ score above 3 over time to reflect the risk of IWS. Then, to generate new hypotheses on optimal weaning strategies in different clinical scenarios, additional simulations were performed of a typical patient's IWS risk over time (i.e., without inclusion of inter-individual variability on baseline IWS severity).

\section{RESULTS}

\section{Clinical Study Data}

In the present study, we included data from 81 children (see Table I), which included $1782 \mathrm{NRS}_{\text {withdrawal }}$ scores collected during a median PICU stay of 16 days (IQR 10$34)$, with $198(11.1 \%)$ of the scores above 3, indicating the presence of IWS. In 42 (52\%) patients, IWS was observed at one or more occasions during the study. The median number of times IWS was observed in a patient was 1 (IQR 0-3); in 13 patients (16\%) IWS was observed six times or more.

The patients received a wide range of cumulative doses of opioids and sedatives (Table I). Some of the studied drugs were given at least once in almost all patients (i.e., morphine (93\%), fentanyl (94\%), midazolam (99\%), and propofol $(84 \%)$ ), while other drugs were given to fewer patients (i.e., ketamine $(60 \%)$, clonidine (44\%), lorazepam (37\%), and methadone (6\%)). Patients received a median of 5 (IQR 4-6) different types of opioids and analgesics, out of the total of 8 opioids and sedatives that were studied here.

\section{Mechanism-Based PKPD Model for Iatrogenic Withdrawal}

To the $\mathrm{NRS}_{\text {withdrawal }}$ data of the patients, we fitted our mechanism-based PKPD model that includes for each drug a hypothetical dependence compartment that equilibrates with the central (plasma) compartment. The concentration in this hypothetical compartment represents the concentration $\left(C_{\text {dependence }}\right)$ that the patient is dependent on at a particular time point, and this is driven by an estimated rate constant $\mathrm{k}_{\mathrm{dep}}$ and the concentration in the plasma compartment (Eq. 1 ), which is in its turn dependent on the drug dosages given over time. When the plasma concentration of a drug is lower than the $C_{\text {dependence }}$, the IWS risk predicted by the model increases with increasing difference between $C_{\text {dependence }}$ and $C_{\text {plasma }}$ (Eq. 2).

Model development started with a base model in which none of the drugs affect IWS, and after development of the 
Table I. Characteristics of the Patients $(n=81)$ Included in This Analysis

\begin{tabular}{|c|c|c|}
\hline Variable & Median (interquartile range) or $n(\%)$ & Range \\
\hline Age (months) & $22(6-75)$ & $1.4-228$ \\
\hline Weight $(\mathrm{kg})$ & $11(6-20)$ & $2.4-70$ \\
\hline Male sex & $41(51)$ & - \\
\hline \multicolumn{3}{|l|}{ Diagnosis category } \\
\hline Respiratory & $32(40)$ & - \\
\hline Cardiac/circulatory & $15(19)$ & - \\
\hline Congenital defects & $1(1)$ & - \\
\hline Surgical/postoperative & $11(14)$ & - \\
\hline Trauma & $7(9)$ & - \\
\hline Other & $15(19)$ & - \\
\hline Length of stay PICU (days) & $16(10-34)$ & $5-96$ \\
\hline Pediatric Risk of Mortality Score III & $9(4-14)$ & $0-30$ \\
\hline \multicolumn{3}{|l|}{ Cumulative drug doses per patient during the study $\mathrm{a}^{\mathrm{a}}$} \\
\hline Morphine $(\mathrm{mg} / \mathrm{kg})$ & $1.5(0.73-2.85)$ & $0-16.3$ \\
\hline Fentanyl $(\mathrm{mcg} / \mathrm{kg})$ & $11(3.5-29)$ & $0-2252$ \\
\hline Midazolam (mg/kg) & $41(23-78)$ & $0-385$ \\
\hline Lorazepam (mg/kg) & $0(0-0.19)$ & $0-27.1$ \\
\hline Ketamine $(\mathrm{mg} / \mathrm{kg})$ & $2.2(0-101)$ & $0-579$ \\
\hline Propofol (mg/kg) & $9.2(2.9-44)$ & $0-837$ \\
\hline Clonidine $(\mathrm{mcg} / \mathrm{kg})$ & $0(0-42.8)$ & $0-1065$ \\
\hline Methadone $(\mathrm{mg} / \mathrm{kg})$ & $0(0-0)$ & $0-51.4$ \\
\hline Number of different opioids and sedatives received ${ }^{b}$ & $5(4-6)$ & $2-8$ \\
\hline
\end{tabular}

$P I C U$ pediatric intensive care unit

${ }^{a}$ Cumulative doses are given as intravenous equivalents. Extravascular doses are transformed into intravenous equivalents using the bioavailability of the population PK models used

${ }^{b}$ Out of the eight opioids and sedatives studied: morphine, fentanyl, midazolam, lorazepam, ketamine, propofol, clonidine, methadone

statistical error model, drugs were added to the model in a stepwise manner (Supplemental Table S1). The dynamics of IWS and dependence could be quantified for three drugs, which significantly improved the fit of the $\mathrm{NRS}_{\text {withdrawal }}$ data: fentanyl $(p<0.001)$, morphine $(p=0.04)$, and ketamine $(p=0.006)$. The estimated values for $k_{\mathrm{dep}}$, the model parameter that controls the rate of dependence development and disappearance over time, varied widely between these drugs, with a high rate constant that indicates fast dependence for fentanyl $\left(k_{\mathrm{dep}}=0.242 \mathrm{~h}^{-1}\right)$ and lower rate constants for morphine and ketamine $\left(k_{\text {dep }}=0.00848 \mathrm{~h}^{-1}\right.$ and $0.0180 \mathrm{~h}^{-1}$, respectively). Midazolam, clonidine, lorazepam, methadone, and propofol were not added to the model, as they did not significantly improve the model fit. No significant influence of patient's age, sex, PRISM III, and diagnosis category was identified $(p>0.01)$. The parameter estimates of the final mechanism-based IWS model are provided in Table II. There was good agreement between the predictions of the model and the observed $\mathrm{NRS}_{\text {withdrawal }}$ data over time in the mirror plots (Fig. 1 a and d) and goodness-of-fit plots (Fig. 1b). Individual fit plots of six randomly selected subjects are provided in Supplemental Material 5.

Figure 2 illustrates that the performance of the final model is in agreement with clinical knowledge on IWS: i.e., abrupt discontinuation of a continuous infusion increases the risk of IWS, but this risk can be reduced by slowly weaning off the continuous infusion $(1,6)$. In each panel of Fig. 2, simulations are shown for a $10-\mathrm{kg}$ patient receiving continuous intravenous morphine infusion of $20 \mathrm{mcg} \mathrm{kg}^{-1} \mathrm{~h}^{-1}$ during 14 days. The morphine concentration in plasma (solid line) quickly reaches steady state in this period, while the concentration that the child has become dependent on ( $C_{\text {dependence }}$, dashed line) slowly increases during the first 14 days. If the morphine infusion is abruptly stopped on day 14 (Fig. 2, left column), there is a large difference between $C_{\text {plasma }}$ and $C_{\text {dependence, }}$, which results in an almost threefold increase in the risk of IWS (bottom row, left column) compared to baseline and this risk decreases over time as $C_{\text {dependence }}$ decreases. By lowering the morphine infusion by $10 \%$ of the initial rate every 24 or $48 \mathrm{~h}$, the $C_{\text {plasma }}$ and $C_{\text {dependence }}$ are lowered more gradually, resulting in lower risk of IWS (Fig. 2, middle and right column). Additionally, a more gradual weaning strategy $(10 \%$ of initial rate every 48 h) results in a slightly lower peak risk of IWS compared to the weaning strategy of reduction with $10 \%$ of initial rate every $24 \mathrm{~h}$.

\section{Weaning Simulations}

We then performed simulations to generate hypotheses on optimal weaning in different scenarios. For simplicity sake, these scenarios only included monotherapy with intravenous administration. Figure 3 illustrates for ketamine the impact of the infusion rate during the 14-day treatment period on the risk of IWS. In all panels, a typical $10-\mathrm{kg}$ child receives continuous ketamine infusion for 14 days, followed by a weaning period. If a 9-day weaning period is used, the IWS risk is higher for a $1.5 \mathrm{mg} \mathrm{kg}^{-1} \mathrm{~h}^{-1}$ infusion (left column) than for a $0.75 \mathrm{mg} \mathrm{kg}^{-1} \mathrm{~h}^{-1}$ infusion (middle column) during the treatment period. When, however, a longer 18-day weaning period (right column) is used after the $1.5 \mathrm{mg} \mathrm{kg}^{-1} \mathrm{~h}^{-1}$ infusion, the risk of IWS is similar to the scenario with a 
Table II. Parameter Estimates of Mechanism-Based Iatrogenic Withdrawal Syndrome (IWS) Model

\begin{tabular}{|c|c|}
\hline Parameter & Estimate $\left(95 \% \mathrm{CI}^{\mathrm{a}}\right)$ \\
\hline \multicolumn{2}{|l|}{ Baseline $\mathrm{NRS}_{\text {withdrawal }}$ (Eq. 3) } \\
\hline Typical baseline & $0.564(0.384-0.828)$ \\
\hline Inter-individual variability baseline (CV\%) & $114 \%(60.6-170)$ \\
\hline \multicolumn{2}{|l|}{ Fentanyl (Eqs. 1 and 2) } \\
\hline$k_{\mathrm{dep}}\left(\mathrm{h}^{-1}\right)$ & $0.242(0.109-0.527)$ \\
\hline Slope $_{\text {fentanyl }}\left(\mathrm{ml} \mathrm{ng}^{-1}\right)$ & $5.93(2.73-12.87)$ \\
\hline \multicolumn{2}{|l|}{ Morphine (Eqs. 1 and 2) } \\
\hline$k_{\text {dep }}\left(\mathrm{h}^{-1}\right)$ & $0.00848(0.00378-0.0190)$ \\
\hline Slope $_{\text {morphine }}\left(\mathrm{ml} \mathrm{ng}^{-1}\right)$ & $0.0687(0.0276-0.171)$ \\
\hline \multicolumn{2}{|l|}{ Ketamine (Eqs. 1 and 2) } \\
\hline$k_{\text {dep }}\left(\mathrm{h}^{-1}\right)$ & $0.0180(0.00668-0.0482)$ \\
\hline Slope $_{\text {ketamine }}\left(\mathrm{ml} \mathrm{mcg}{ }^{-1}\right)$ & $1.59(0.768-3.29)$ \\
\hline \multicolumn{2}{|l|}{ Overdispersion and Markov probability inflation } \\
\hline$\delta$ & $0.428(0.350-0.506)$ \\
\hline$\pi 0 \mid 0$ & $0.401(0.321-0.486)$ \\
\hline$\pi 0 \mid \mathrm{x}$ & $0.216(0.179-0.258)$ \\
\hline$\pi \pm 1=\pi \pm 2$ & $0.0698(0.0422-0.113)^{\mathrm{b}}$ \\
\hline
\end{tabular}

$C I$ confidence interval; $\mathrm{NRS}_{\text {withdrawal, }}$ score of withdrawal severity on a numerical rating scale; $\mathrm{CV} \%$, coefficient of variation; $\delta$, coefficient of dispersion parameter; $\pi 0 \mid 0$, probability inflation of observing the same score as before if the previous score was zero; $\pi 0 \mid \mathrm{x}$, probability inflation of observing the same score as before if the previous score was not zero; $\pi \pm 1$, probability inflation of observing a score that is 1 point higher or lower than the previous score; $\pi \pm 2$, probability inflation of observing a score that is 2 points higher or lower than the previous score ${ }_{b}^{a}$ Calculated from standard error of estimates generated from NONMEM's covariance step

${ }^{b} \pi \pm 1$ and $\pi \pm 2$ were constrained to be equal to each other

a

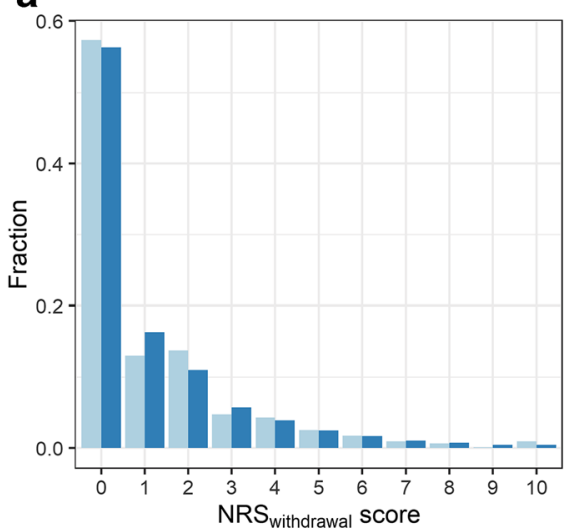

b

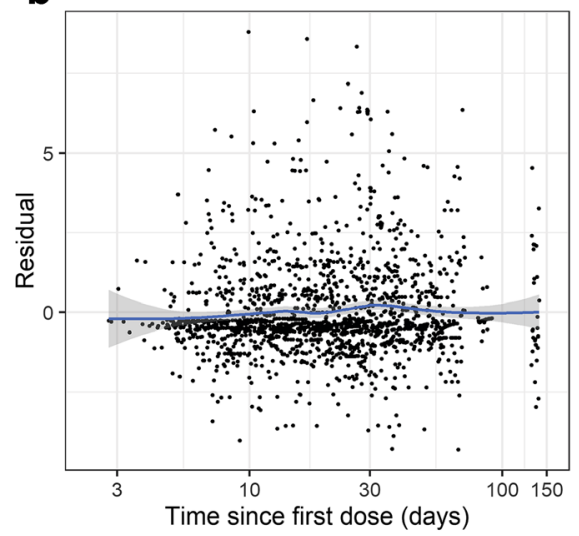

c

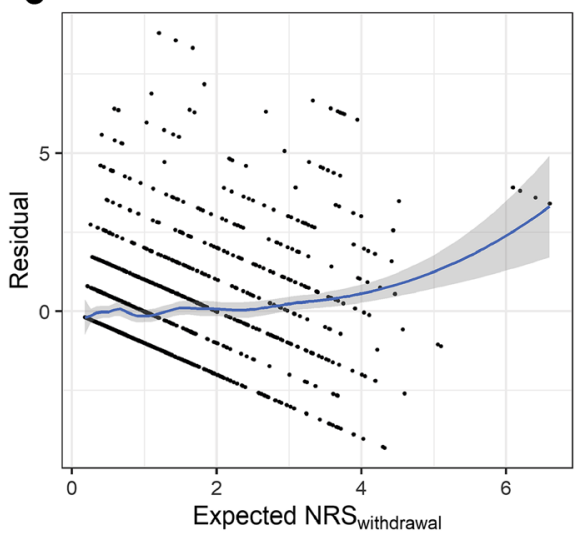

d

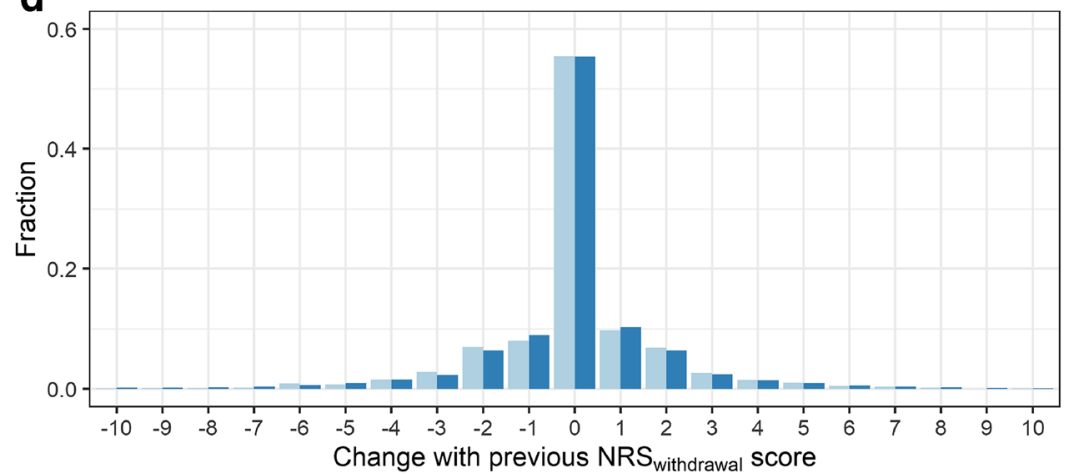

Fig. 1. Model diagnostic plots of final PKPD model. a and d Mirror plot of observed (light blue) and predicted (dark blue) frequencies of $\mathrm{NRS}_{\text {withdrawal }}$ scores a and their transitions between consecutive observations $\mathbf{d}$. The predicted frequencies are calculated as the mean of the individual post hoc estimates of the probabilities at each observation. Transition is calculated as follows: current $\mathrm{NRS}_{\text {withdrawal }}$ score - previous $\mathrm{NRS}_{\text {withdrawal }}$ score. $\mathbf{b}$ and $\mathbf{c}$ Each point represents one observation, where the residual is calculated as the difference between the observed

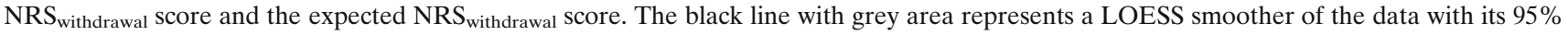
confidence interval, respectively 
No weaning
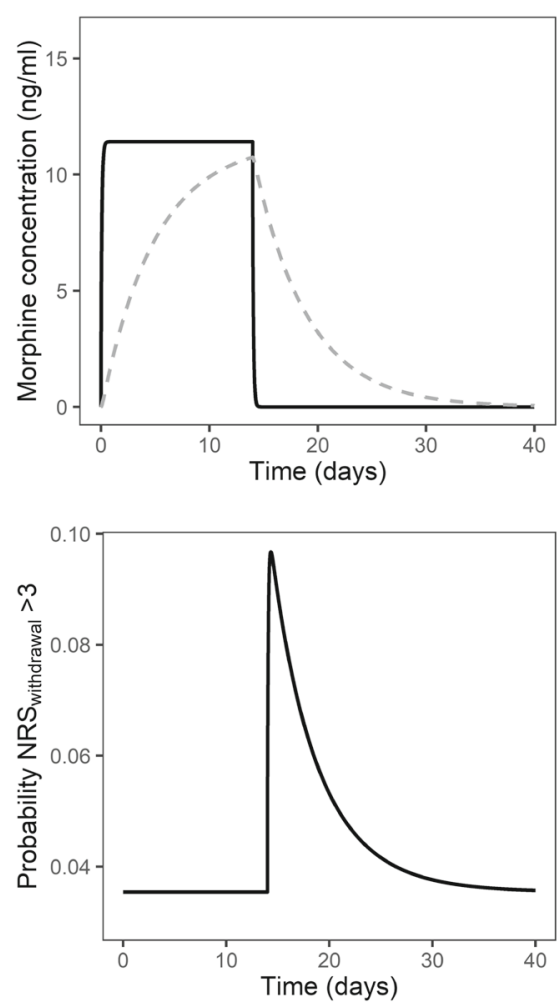

$-10 \%$ every 24 hours
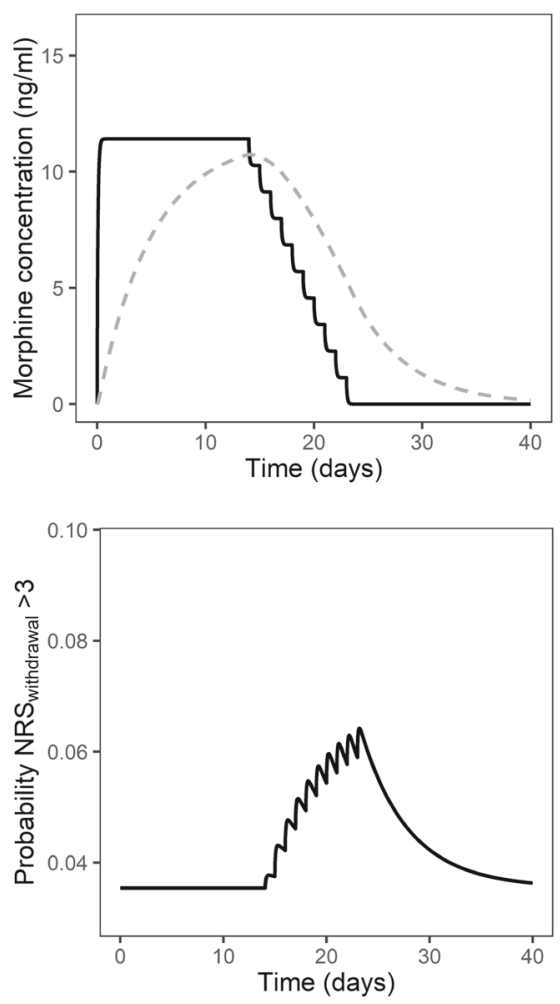

$-10 \%$ every 48 hours
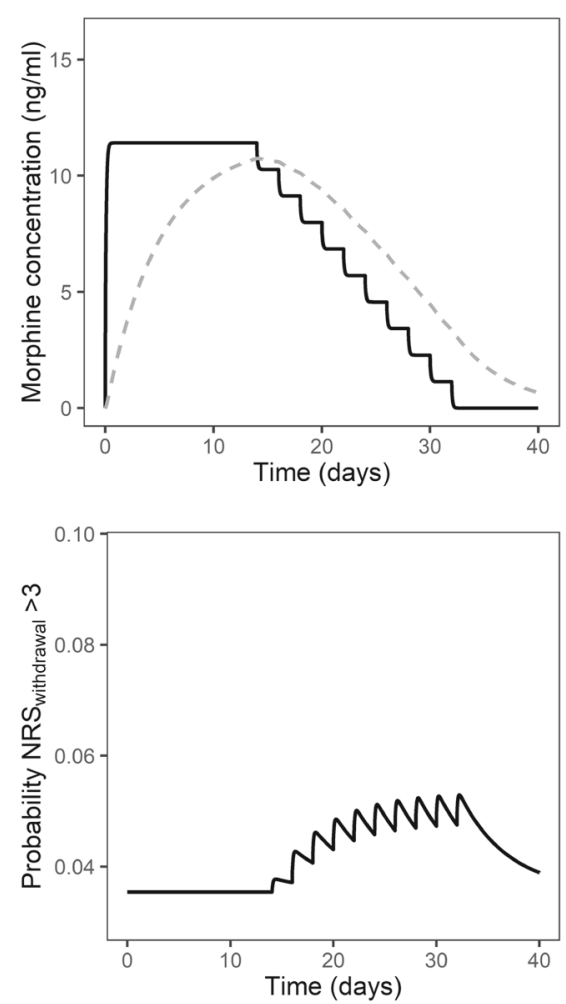

Fig. 2. Illustration of the risk of iatrogenic withdrawal syndrome (IWS) over time predicted by the mechanism-based IWS model for different weaning strategies after 14-day treatment period with continuous intravenous morphine at $20 \mathrm{mcg} \mathrm{kg}^{-1} \mathrm{~h}^{-1}$ in a typical patient with a $10-\mathrm{kg}$ body weight. The top row panels show the simulated morphine concentrations in plasma ( $C_{\text {plasma }}$, solid black line) and morphine concentrations that the child has become dependent on $\left(C_{\text {dependence, }}\right.$ dashed grey line). The bottom row panels show the predicted probability of an $\mathrm{NRS}_{\text {withdrawal }}$ score above 3, which indicates IWS

lower infusion rate but a shorter 9-day weaning period. The same phenomenon occurs in simulations with morphine and fentanyl (Supplemental Material 6 and 7, respectively)

Figure 4 compares for fentanyl the impact on IWS risk of using frequent, small weaning steps versus less frequent, but greater weaning steps. In all scenarios shown in Fig. 4, a typical $10-\mathrm{kg}$ child receives 14 days of continuous intravenous fentanyl at $1.5 \mathrm{mcg} \mathrm{kg}^{-1} \mathrm{~h}^{-1}$, followed by an 8-day period of weaning. Due to the high value of $\mathrm{k}_{\mathrm{dep}}$ of fentanyl, $C_{\text {plasma }}$ and $C_{\text {dependence }}$ closely follow each other; therefore, the risk of IWS is primarily associated with the rate of decrease in $C_{\text {plasma. }}$ This figure illustrates that due to these IWS characteristics, fentanyl is best weaned off in small steps to avoid peak risks of IWS. When fentanyl is weaned off in steps of $20 \%$ of the initial rate every $48 \mathrm{~h}$ (right column), there is an over twofold increase in the peak risk of IWS. The peak risk of IWS is seen when the infusion rate is decreased, as that is when the rate of decrease in $C_{\text {plasma }}$ is highest. A lower risk of IWS can be expected when applying smaller, but more frequent (every 12 or $24 \mathrm{~h}$ ) reductions in the fentanyl infusion rate (Fig. 4, left and middle column). This phenomenon is not seen in simulations with morphine, as this drug has a much lower $k_{\mathrm{dep}}$, and the simulated risk of IWS in scenarios in which morphine is weaned in steps of $48 \mathrm{~h}$ is similar to that in scenarios with weaning in steps of $12 \mathrm{~h}$ (Supplemental Material 8).

\section{DISCUSSION}

We currently lack a quantitative understanding of how different weaning strategies can affect the risk of IWS over time in children who required prolonged treatment of (combinations of) opioids and/or sedatives. To allow prediction of the risk of IWS over time, we applied a new mechanism-based PKPD modeling approach in a PICU population of children older than 1 month (median age 22 months), who received continuous infusions with opioids and/ or sedatives for 5 days or more. The model adequately fitted the observed $\mathrm{NRS}_{\text {withdrawal }}$ data in this population and quantified the IWS dynamics of fentanyl, morphine, and ketamine. Additionally, we confirmed that the predictions of the model are in agreement with what clinical knowledge dictates: abrupt discontinuation of a continuous infusion will result in an increased risk of IWS, but this risk can be reduced by slowly weaning off the continuous infusion (Fig. 2) $(1,6)$.

Additional simulations generated new hypotheses that could guide future clinical research on optimal weaning strategies. For the three drugs included in the IWS model, simulations suggested that a longer weaning period should be used when a higher infusion rate is administered before weaning, to have the same IWS risk as patients with a lower infusion rate (Fig. 3, Supplemental Material 6 and 7). Similarly, these results suggest that patients with a relatively 


\section{Ketamine}

\section{9 day weaning from $1.5 \mathrm{mg} \mathrm{kg}^{-1} \mathrm{hr}^{-1}$}
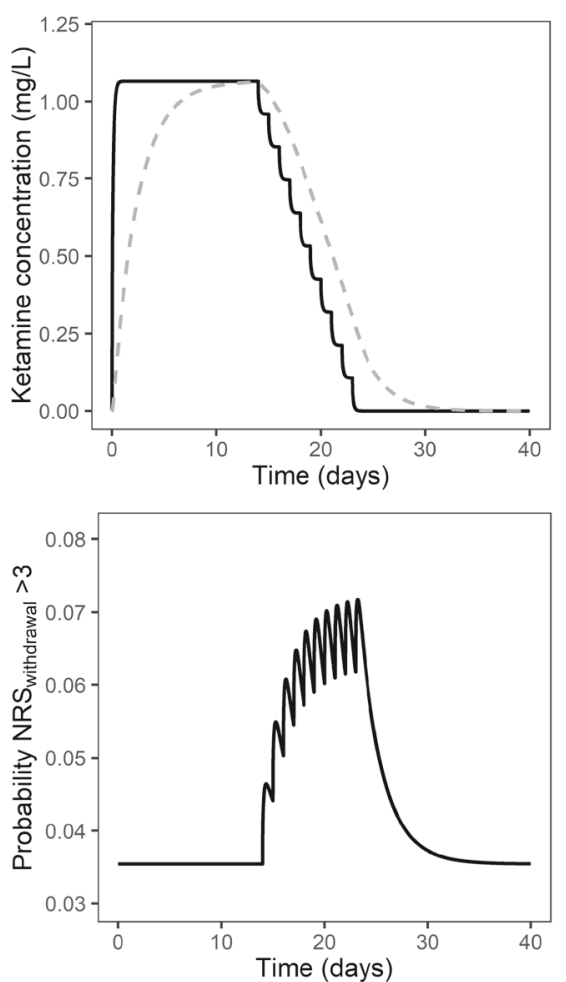

9 day weaning from

$0.75 \mathrm{mg} \mathrm{kg}^{-1} \mathrm{hr}^{-1}$
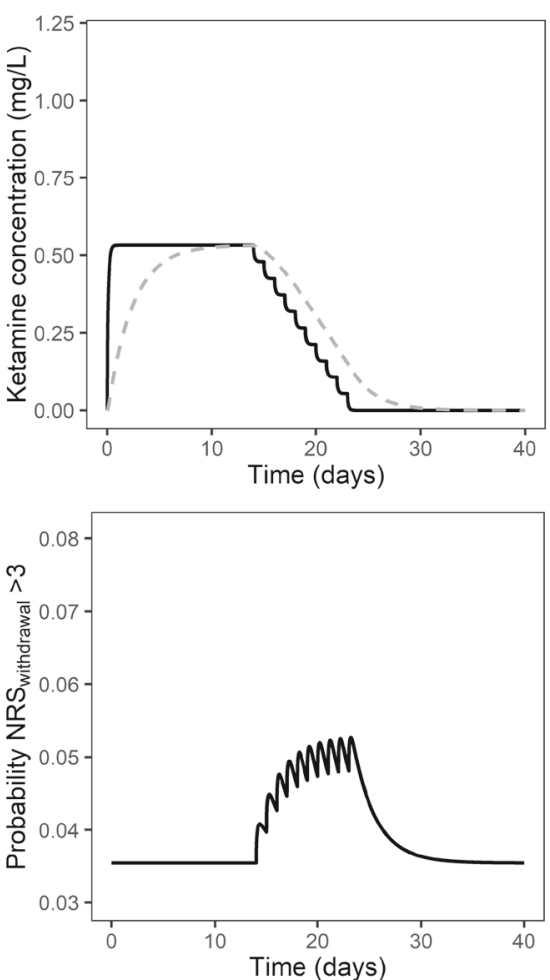

18 day weaning from $1.5 \mathrm{mg} \mathrm{kg}^{-1} \mathrm{hr}^{-1}$
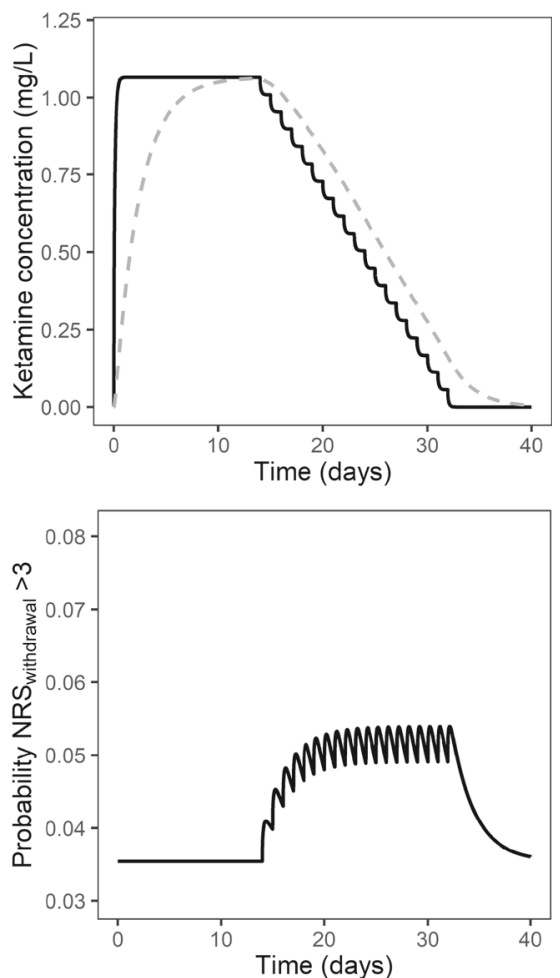

Fig. 3. The impact of the ketamine infusion rate $\left(1.5\right.$ or $\left.0.75 \mathrm{mg} \mathrm{kg}^{-1} \mathrm{~h}^{-1}\right)$ during a 14-day treatment period and weaning duration (9- or 18-day weaning) on the risk of iatrogenic withdrawal syndrome (IWS) during weaning in a typical patient with a 10-kg body weight. The top row shows the simulated ketamine concentrations in plasma ( $C_{\text {plasma }}$, solid black line) and ketamine concentrations that the child has become

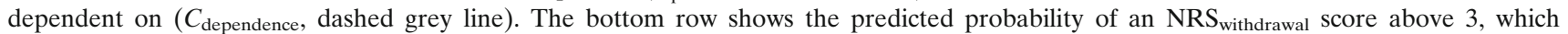
indicates IWS. In all scenarios simulated here, the time between consecutive weaning steps is $24 \mathrm{~h}$

low infusion rate might be weaned relatively fast without a high risk of IWS. While these results may seem intuitive, they contradict with most weaning protocols reported in literature. Most weaning protocols tend to stepwise reduce the infusion rate by a set percentage of the original infusion rate, resulting in the same planned weaning period for all patients (e.g., $10 \%$ reduction every $24 \mathrm{~h})(5,6,36)$.

Regarding fentanyl, from finding that there is a fast rate of dependence development and disappearance, we hypothesize that small frequent (e.g., every $12 \mathrm{~h}$ ) reductions of the infusion rate could reduce the IWS risk of fentanyl compared to greater but less frequent (e.g., every $48 \mathrm{~h}$ ) reductions. Interestingly, weaning from morphine is less dependent on the intervals between weaning steps, as the predicted IWS risk from morphine was similar in scenarios with weaning every 12 or $48 \mathrm{~h}$ (Supplemental Material 8). These observations might explain clinical observations that the risk of IWS is higher for patients treated with fentanyl than for patients treated with morphine, as the weaning strategy of fentanyl would be less forgiving to sudden large reductions in infusion rate (3, 37-39). Our results suggest that a "small but frequent" approach to fentanyl weaning might reduce the heightened risk for IWS after fentanyl treatment.
Through the simulations discussed here, the model allowed us to explore weaning scenarios that would be not be possible to prospectively study in real patients due to ethical reasons, e.g., sticking to a strict monotherapy treatment and weaning protocol without allowing for any dose adjustments upon medical need. These simulation scenarios demonstrate the utility of the PKPD modeling approach to explore the interplay between pre-weaning drug treatment, weaning strategy, and the risk of IWS. In this paper, for the sake of simplicity, we only showed simulations in which a single drug is responsible for IWS, but the model can also be used to predict the risk of IWS in scenarios with multiple drugs.

We found a large difference in the estimated $k_{\text {dep }}$ between fentanyl and morphine, although both drugs target the mu-opioid receptor (40). It has been suggested that some differences in pharmacology between opioids originate from a complex interplay between drug distribution in the body and their differential activation profiles of different mu opioid receptor subtypes, which might also explain the differences in $k_{\text {dep }}$ we observed here $(40,41)$. Another possible explanation of the difference between fentanyl and morphine is that morphine-3-glucoronide and morphine-6-glucoronide, which 
Fentanyl

\section{Small weaning steps every 12 hours}
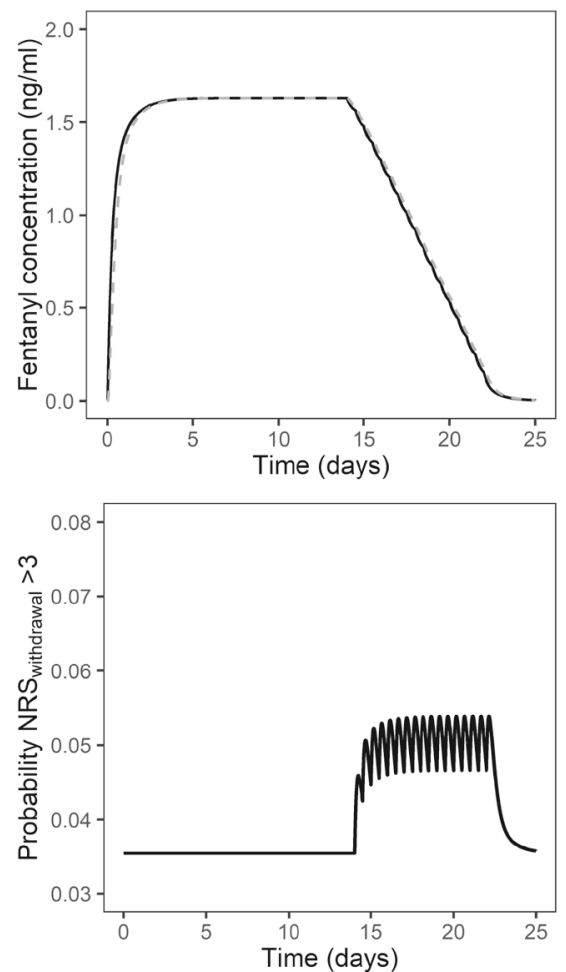

Medium weaning steps every 24 hours
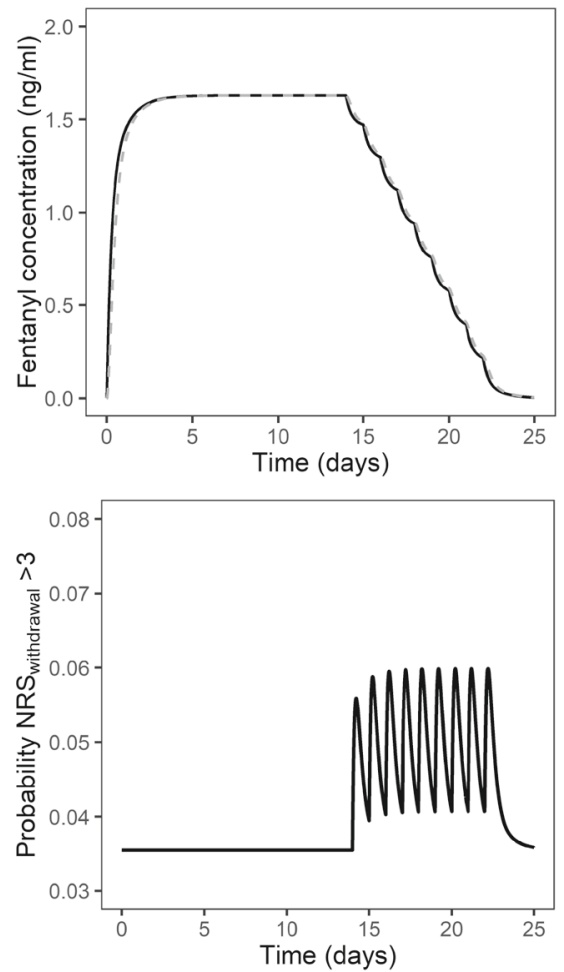

\section{Large weaning steps every 48 hours}
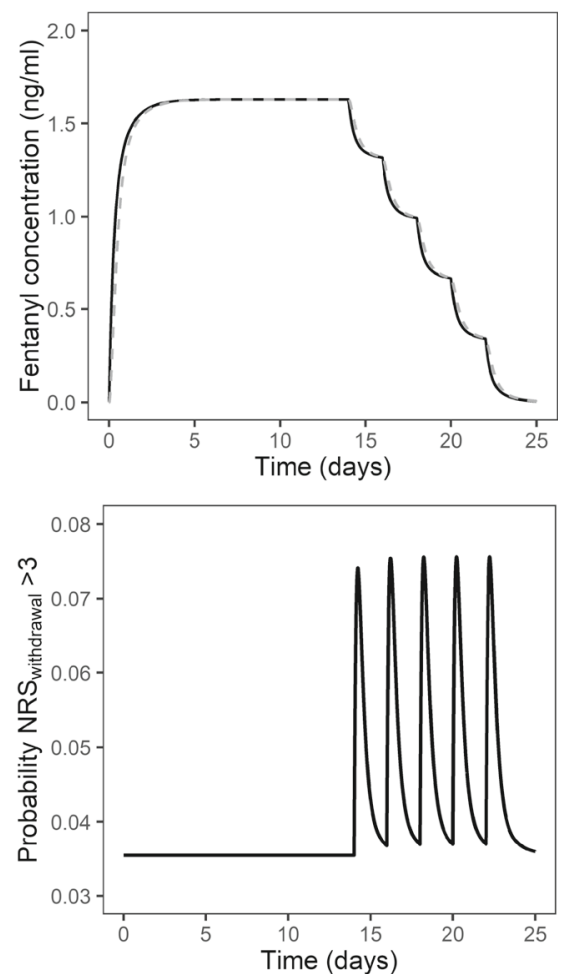

Fig. 4. The impact of time between weaning steps and weaning step size on the risk of iatrogenic withdrawal syndrome (IWS) after 14-day treatment period with continuous intravenous fentanyl at $1.5 \mathrm{mcg} \mathrm{kg}^{-1} \mathrm{~h}^{-1}$ in a typical patient with a $10-\mathrm{kg}$ body weight. In all three scenarios, the time between the first reduction in the fentanyl infusion and the complete discontinuations of the fentanyl infusion is 8 days; the scenarios only vary in time between weaning steps and the infusion rate reduction in these steps. The top row shows the simulated fentanyl concentrations in plasma (solid black line) and fentanyl concentrations that the child has become dependent on (dashed grey line). Due to the high dependence rate of fentanyl $\left(k_{\text {dep }}=0.265 \mathrm{~h}^{-1}\right), C_{\text {plasma }}$ and $C_{\text {dependence }}$ closely follow each other. The bottom row shows the predicted probability of an $\mathrm{NRS}_{\text {withdrawal }}$ score above 3, which indicates IWS

are active metabolites of morphine that were not incorporated in the model, may have contributed to the observed IWS dynamics after morphine treatment (40). This might be especially relevant in patients with impaired renal function as this could result in a long half-life and accumulation of these metabolites.

Among the patients analyzed in the current study, 52\% had one or more observations of IWS. The reported incidence of IWS in literature varies widely (5-87\%), which likely reflects the heterogeneity of both the patient population and the clinical protocols used for both sedation and weaning in different hospitals. Differences in these protocols may impact the applicability of our findings to other clinical settings. Another limitation of the study is the lack of simulation-based diagnostics, which are considered the gold standard model evaluation techniques, but which could not be created because the complex dosing patterns (with up- and downtitration of multiple drugs depending on $\mathrm{NRS}_{\text {withdrawal }}$ scores) could not be re-created in a simulation (42).

Although we could quantify the impact of three drugs on IWS in children, we cannot exclude a confounding effect from drugs not included in the model because of a lack of statistical significance. These include drugs that might contribute to IWS (e.g., sedatives like midazolam) or drugs used to manage IWS during weaning (e.g., clonidine, methadone) (5). The effects of these drugs on IWS in this study cohort may have been more modest or more variable in nature than the effects of the drugs included in the model. Additionally, the relatively low occurrence of high $\mathrm{NRS}_{\text {withdrawal }}$ scores in this study cohort limits the statistical power to identify the effects of additional drugs on IWS. By applying our mechanismbased modeling approach in larger datasets, we might obtain a more comprehensive picture of the relative influences of these drugs on IWS and improve the predictive performance of the model. This also includes drugs, like dexmedetomidine, that were not used in this study, but which may also induce IWS after prolonged use (43).

\section{CONCLUSIONS}

We developed a novel mechanism-based PKPD modeling approach to quantify the IWS dynamics of morphine, fentanyl, and ketamine from a large clinical dataset of critically ill children. The simulations performed with this 
model suggest that with a higher infusion rate before weaning a longer weaning period would be needed to have the same IWS risk as patients with a lower infusion rate. For fentanyl specifically, weaning with small but frequent reductions in the infusion rate might reduce the heightened risk for IWS after fentanyl treatment. The mechanism-based modeling approach introduced here might well be applied in other datasets to quantify the IWS dynamics of other opioids and sedatives.

\section{SUPPLEMENTARY INFORMATION}

The online version contains supplementary material available at https://doi.org/10.1208/s12248-021-00586-w.

\section{ACKNOWLEDGEMENTS}

The authors thank Ko Hagoort for editing the manuscript.

Open Access This article is licensed under a Creative Commons Attribution 4.0 International License, which permits use, sharing, adaptation, distribution and reproduction in any medium or format, as long as you give appropriate credit to the original author(s) and the source, provide a link to the Creative Commons licence, and indicate if changes were made. The images or other third party material in this article are included in the article's Creative Commons licence, unless indicated otherwise in a credit line to the material. If material is not included in the article's Creative Commons licence and your intended use is not permitted by statutory regulation or exceeds the permitted use, you will need to obtain permission directly from the copyright holder. To view a copy of this licence, visit http://creativecommons.org/licenses/by/4.0/.

\section{REFERENCES}

1. Tobias JD. Tolerance, withdrawal, and physical dependency after long-term sedation and analgesia of children in the pediatric intensive care unit. Crit Care Med. 2000;28:2122-32.

2. Ista E, van Dijk M, de Hoog M, Tibboel D, Duivenvoorden HJ. Construction of the Sophia Observation withdrawal Symptomsscale (SOS) for critically ill children. Intensive Care Med. 2009;35:1075-81. https://doi.org/10.1007/s00134-009-1487-3.

3. Best KM, Boullata JI, Curley MA. Risk factors associated with iatrogenic opioid and benzodiazepine withdrawal in critically ill pediatric patients: a systematic review and conceptual model. Pediatr Crit Care Med. 2015;16:175-83. https://doi.org/10.1097/ PCC. 0000000000000306 .

4. Goulooze SC, Ista E, van Dijk M, Hankemeier T, Tibboel D, Knibbe CAJ, et al. Supervised multidimensional item response theory modeling of pediatric iatrogenic withdrawal symptoms. CPT Pharmacometrics Syst Pharmacol. 2019;8:904-12. https:// doi.org/10.1002/psp4.12469.

5. Vipond JM, Heiberger AL, Thompson PA, Huber JN. Shortened taper duration after implementation of a standardized protocol for iatrogenic benzodiazepine and opioid withdrawal in pediatric patients: results of a cohort study. Pediatr Qual Saf. 2018;3:e079. https://doi.org/10.1097/pq9.0000000000000079.

6. Fenn NE 3rd, Plake KS. Opioid and benzodiazepine weaning in pediatric patients: review of current literature. Pharmacotherapy. 2017;37:1458-68. https://doi.org/10.1002/phar.2026.

7. Upton RN, Mould DR. Basic concepts in population modeling, simulation, and model-based drug development: part 3- introduction to pharmacodynamic modeling methods. CPT Pharmacometrics Syst Pharmacol. 2014;3:e88. https://doi.org/ 10.1038/psp.2013.71.

8. Harris J, Ramelet AS, van Dijk M, Pokorna P, Wielenga J, Tume L, et al. Clinical recommendations for pain, sedation, withdrawal and delirium assessment in critically ill infants and children: an ESPNIC position statement for healthcare professionals. Intensive Care Med. 2016;42:972-86. https://doi.org/ 10.1007/s00134-016-4344-1.

9. Ista E, de Hoog M, Tibboel D, Duivenvoorden HJ, van Dijk M. Psychometric evaluation of the Sophia Observation withdrawal symptoms scale in critically ill children. Pediatr Crit Care Med. 2013;14:761-9. https://doi.org/10.1097/PCC.0b013e31829f5be1.

10. Vet NJ, Brussee JM, de Hoog M, Mooij MG, Verlaat CW, Jerchel IS, et al. Inflammation and organ failure severely affect midazolam clearance in critically ill children. Am J Respir Crit Care Med. 2016;194:58-66. https://doi.org/10.1164/rccm.201510-2114OC.

11. Wang C, Sadhavisvam S, Krekels EH, Dahan A, Tibboel D, Danhof M, et al. Developmental changes in morphine clearance across the entire paediatric age range are best described by a bodyweight-dependent exponent model. Clin Drug Investig. 2013;33:523-34. https://doi.org/10.1007/s40261-013-0097-6.

12. Van Driest SL, Marshall MD, Hachey B, Beck C, Crum K, Owen J, et al. Pragmatic pharmacology: population pharmacokinetic analysis of fentanyl using remnant samples from children after cardiac surgery. Br J Clin Pharmacol. 2016;81:1165-74. https://doi.org/10.1111/bcp.12903.

13. Choi L, Ferrell BA, Vasilevskis EE, Pandharipande PP, Heltsley R, Ely EW, et al. Population pharmacokinetics of fentanyl in the critically ill. Crit Care Med. 2016;44:64-72. https://doi.org/ 10.1097/CCM.0000000000001347.

14. Potts AL, Larsson P, Eksborg S, Warman G, Lonnqvist PA, Anderson BJ. Clonidine disposition in children; a population analysis. Paediatr Anaesth. 2007;17:924-33. https://doi.org/ 10.1111/j.1460-9592.2007.02251.x.

15. Rigby-Jones AE, Nolan JA, Priston MJ, Wright PM, Sneyd JR, Wolf AR. Pharmacokinetics of propofol infusions in critically ill neonates, infants, and children in an intensive care unit. Anesthesiology. 2002;97:1393-400. https://doi.org/10.1097/ 00000542-200212000-00010.

16. Elkomy MH, Drover DR, Hammer GB, Galinkin JL, Ramamoorthy C. Population pharmacokinetics of ketamine in children with heart disease. Int J Pharm. 2015;478:223-31. https://doi.org/10.1016/j.ijpharm.2014.11.026.

17. Ward RM, Drover DR, Hammer GB, Stemland CJ, Kern S, Tristani-Firouzi M, et al. The pharmacokinetics of methadone and its metabolites in neonates, infants, and children. Paediatr Anaesth. 2014;24:591-601. https://doi.org/10.1111/pan.12385.

18. Swart EL, Zuideveld KP, de Jongh J, Danhof M, Thijs LG, Strack van Schijndel RM. Comparative population pharmacokinetics of lorazepam and midazolam during long-term continuous infusion in critically ill patients. Br J Clin Pharmacol. 2004;57:135-45. https://doi.org/10.1046/j.1365-2125.2003.01957.x.

19. Gonzalez D, Chamberlain JM, Guptill JT, Cohen-Wolkowiez M, Harper B, Zhao J, et al. Population pharmacokinetics and exploratory pharmacodynamics of lorazepam in pediatric status epilepticus. Clin Pharmacokinet. 2017;56:941-51. https://doi.org/ 10.1007/s40262-016-0486-0.

20. Calvier EA, Krekels EH, Valitalo PA, Rostami-Hodjegan A, Tibboel D, Danhof M, et al. Allometric scaling of clearance in paediatric patients: when does the magic of 0.75 fade? Clin Pharmacokinet. 2017;56:273-85. https://doi.org/10.1007/s40262016-0436-x.

21. Payne K, Mattheyse FJ, Liebenberg D, Dawes T. The pharmacokinetics of midazolam in paediatric patients. Eur J Clin Pharmacol. 1989;37:267-72. https://doi.org/10.1007/bf00679782.

22. Larsson P, Nordlinder A, Bergendahl HT, Lonnqvist PA, Eksborg S, Almenrader N, et al. Oral bioavailability of clonidine in children. Paediatr Anaesth. 2011;21:335-40. https://doi.org/10.1111/j.1460-9592.2010.03397.x.

23. Brunette KE, Anderson BJ, Thomas J, Wiesner L, Herd DW, Schulein S. Exploring the pharmacokinetics of oral ketamine in children undergoing burns procedures. Paediatr Anaesth. 2011;21:653-62. https://doi.org/10.1111/j.1460-9592.2011.03548.x. 
24. Moolenaar F, Visser J, Leuverman A, Schoonen BJM. Bioavailability of morphine from suppositories. Int J Pharm. 1988;45:161-4. https://doi.org/10.1016/0378-5173(88)90046-4.

25. Hunt A, Joel S, Dick G, Goldman A. Population pharmacokinetics of oral morphine and its glucuronides in children receiving morphine as immediate-release liquid or sustainedrelease tablets for cancer pain. J Pediatr. 1999;135:47-55. https:// doi.org/10.1016/s0022-3476(99)70326-4.

26. Jonsson T, Christensen CB, Jordening H, Frolund C. The bioavailability of rectally administered morphine. Pharmacol Toxicol. 1988;62:203-5. https://doi.org/10.1111/j.16000773.1988.tb01872.x

27. Gorlin AW, Rosenfeld DM, Maloney J, Wie CS, McGarvey J, Trentman TL. Survey of pain specialists regarding conversion of high-dose intravenous to neuraxial opioids. J Pain Res. 2016;9:693-700. https://doi.org/10.2147/Jpr.S113216.

28. Schwagmeier R, Alincic S, Striebel HW. Midazolam pharmacokinetics following intravenous and buccal administration. Br J Clin Pharmacol. 1998;46:203-6. https://doi.org/10.1046/j.13652125.1998.00781.x.

29. Bjorkman S, Rigemar G, Idvall J. Pharmacokinetics of midazolam given as an intranasal spray to adult surgical patients. Br J Anaesth. 1997;79:575-80. https://doi.org/10.1093/bja/79.5.575.

30. Dale O, Sheffels P, Kharasch ED. Bioavailabilities of rectal and oral methadone in healthy subjects. $\mathrm{Br} \mathrm{J}$ Clin Pharmacol. 2004;58:156-62. https://doi.org/10.1111/j.1365-2125.2004.02116.x.

31. Greenblatt DJ, Shader RI, Franke K, MacLaughlin DS, Harmatz JS, Allen MD, et al. Pharmacokinetics and bioavailability of intravenous, intramuscular, and oral lorazepam in humans. J Pharm Sci. 1979;68:57-63. https://doi.org/10.1002/ jps.2600680119.

32. Poulain P, Hoskin PJ, Hanks GW, O AO, Walker VA, Johnston A, et al. Relative bioavailability of controlled release morphine tablets (MST continus) in cancer patients. $\mathrm{Br} \mathrm{J}$ Anaesth. 1988;61:569-74. https://doi.org/10.1093/bja/61.5.569.

33. Plan EL, Elshoff JP, Stockis A, Sargentini-Maier ML, Karlsson MO. Likert pain score modeling: a Markov integer model and an autoregressive continuous model. Clin Pharmacol Ther. 2012;91:820-8. https://doi.org/10.1038/clpt.2011.301.

34. Bauer RJ. NONMEM Tutorial Part II: Estimation methods and advanced examples. CPT Pharmacometrics Syst Pharmacol. 2019;8:538-56. https://doi.org/10.1002/psp4.12422.
35. Ueckert S. Modeling composite assessment data using item response theory. CPT Pharmacometrics Syst Pharmacol. 2018;7:205-18. https://doi.org/10.1002/psp4.12280.

36. Solodiuk JC, Greco CD, O'Donnell KA, Morrill DR, Curley MAQ. Effect of a sedation weaning protocol on safety and medication use among hospitalized children post critical illness. J Pediatr Nurs. 2019;49:18-23. https://doi.org/10.1016/ j.pedn.2019.08.001.

37. Franck LS, Naughton I, Winter I. Opioid and benzodiazepine withdrawal symptoms in paediatric intensive care patients. Intensive Crit Care Nurs. 2004;20:344-51. https://doi.org/ 10.1016/j.iccn.2004.07.008.

38. Franck LS, Vilardi J, Durand D, Powers R. Opioid withdrawal in neonates after continuous infusions of morphine or fentanyl during extracorporeal membrane oxygenation. Am J Crit Care. 1998;7:364-9.

39. Amigoni A, Mondardini MC, Vittadello I, Zaglia F, Rossetti E, Vitale F, et al. Withdrawal Assessment Tool-1 Monitoring in PICU: a multicenter study on iatrogenic withdrawal syndrome. Pediatr Crit Care Med. 2017;18:e86-91. https://doi.org/10.1097/ PCC.0000000000001054.

40. Drewes AM, Jensen RD, Nielsen LM, Droney J, Christrup LL, Arendt-Nielsen L, et al. Differences between opioids: pharmacological, experimental, clinical and economical perspectives. $\mathrm{Br}$ J Clin Pharmacol. 2013;75:60-78. https://doi.org/10.1111/j.13652125.2012.04317.x.

41. Pasternak GW. Molecular insights into mu opioid pharmacology: from the clinic to the bench. Clin J Pain. 2010;26(Suppl 1):S3-9. https://doi.org/10.1097/AJP.0b013e3181c49d2e.

42. Karlsson MO, Savic RM. Diagnosing model diagnostics. Clin Pharmacol Ther. 2007;82:17-20. https://doi.org/10.1038/ sj.clpt.6100241.

43. Haenecour AS, Seto W, Urbain CM, Stephens D, Laussen PC, Balit CR. Prolonged dexmedetomidine infusion and drug withdrawal in critically ill children. J Pediatr Pharmacol Ther. 2017;22:453-60. https://doi.org/10.5863/1551-6776-22.6.453.

Publisher's Note Springer Nature remains neutral with regard to jurisdictional claims in published maps and institutional affiliations. 